\title{
Temporal analysis of individual ethanol consumption in socially housed mice and the effects of oxytocin
}

\author{
Maya A. Caruso ${ }^{1} \cdot$ Meridith T. Robins $^{1} \cdot$ Hannah D. Fulenwider ${ }^{1} \cdot$ Andrey E. Ryabinin $^{1}$ (D) \\ Received: 3 June 2020 / Accepted: 1 December 2020 / Published online: 6 January 2021 \\ (C) The Author(s), under exclusive licence to Springer-Verlag GmbH, DE part of Springer Nature 2021
}

\begin{abstract}
Rationale The majority of preclinical studies assessing treatments for alcohol use disorder use singly housed animals. Because social factors affect ethanol intake, studies investigating such treatments in group-housed animals are needed.

Objectives We investigated the effects of repeated oxytocin treatment on ethanol intake in socially housed male and female C57BL/6J mice.

Methods We used the novel "Herdsman" system implementing radiotracking technology to measure individual ethanol intake in group-housed animals. Mice were housed in same-sex groups of 4 per cage and exposed to 3 and $6 \%$ ethanol solutions. After baseline drinking was established, half of the animals in each cage received repeated intraperitoneal injections of $3 \mathrm{mg} / \mathrm{kg}$ oxytocin.

Results During baseline, females consumed more ethanol than males partly due to greater number of ethanol drinks taken by females. We also observed a gradual development of two peaks of ethanol consumption during the dark phase of the circadian cycle. The effects of oxytocin treatment were short-acting and varied across treatment days. Oxytocin significantly decreased ethanol intake on three out the four treatment days. On the fourth treatment day, oxytocin decreased ethanol intake and water intake.

Conclusion The greater intake of ethanol in female mice is associated with the number of drinks taken. Oxytocin treatments not only cause an acute decrease in ethanol consumption, but can also change in efficacy over time. While the oxytocin system remains a promising therapeutic target for alcoholism, studies investigating longer periods of repeated oxytocin treatment and those using additional oxytocin receptor agonists are warranted.
\end{abstract}

Keywords Alcohol · HM2 - Radiofrequency identification · RFID · Oxytocin · Two-bottle choice - Socially housed · Sex differences $\cdot$ Ethanol preference $\cdot$ Drink size

\section{Introduction}

Alcohol use disorder (AUD) affects approximately $5.8 \%$ of the US population (National Institute on Alcohol Abuse and Alcoholism 2018). The development of this disorder involves the transition from a state of recreational alcohol use to that of an uncontrolled pattern of intake (Koob and Volkow 2016), and eventually, if untreated, can lead to compulsive use and the emergence of a negative affective state when abstaining

Andrey E. Ryabinin

ryabinin@ohsu.edu

1 Department of Behavioral Neuroscience, Oregon Health \& Science University, 3181 SW Sam Jackson Park Road L470,

Portland, OR 97239, USA from alcohol use (National Institute on Alcohol Abuse and Alcoholism 2018). There are currently three drugs approved for treatment of AUD by the US Food and Drug Administration (FDA): naltrexone, acamprosate, and disulfiram (National Institute on Alcohol Abuse and Alcoholism 2014). While these medications reduce alcohol consumption in certain patient populations, the overall effectiveness of these treatments varies (Swift and Aston 2015; Akbar et al. 2018). Thus, the identification of additional therapeutic targets is greatly needed.

Oxytocin (OXT) is a nine-amino acid peptide that has been linked to a variety of physiological functions and behaviors such as lactation, parturition, and pair and social bonding (Williams et al. 1994; Kramer et al. 2006; Lee et al. 2016, 2009). Recently, though, OXT has also gained interest as a potential treatment for substance use disorders (Neumann 
et al. 2013; Lee et al. 2016). A growing amount of preclinical and clinical data has demonstrated the ability of OXT to reduce alcohol intake using intranasal, intravenous, intraperitoneal (IP), and intracranial routes of administration (McGregor and Bowen 2012; Peters et al. 2013; MacFadyen et al. 2016; King et al. 2017; Stevenson et al. 2017; Hansson et al. 2018; Tunstall et al. 2019; Walcott and Ryabinin 2020). However, a number of questions regarding the effectiveness and specificity of OXT treatment for this behavior remain. In particular, it is not clear whether OXT's effects on alcohol intake change over time and/or as a result of repeated treatment. The latter issue is of particular importance, as it is expected that patients with AUD would be treated with OXT repeatedly, and acute versus repeated OXT exposures have been shown to have different effects on various behaviors (Szabó et al. 1985; Bowen et al. 2011; Lee et al. 2016; Benner et al. 2018). Thus, as most of the previously described studies have only examined the effects of OXT following a single treatment, a primary goal of this experiment was to investigate the effects of several OXT treatments on ethanol consumption in male and female mice.

To assess the effects of pharmacological intervention on voluntary ethanol intake, researchers often use a traditional two-bottle choice model where single-housed rodents have access to one bottle of ethanol and one bottle of water (Crabbe et al. 2011; Becker 2013; Huynh et al. 2019). Single-housing rodents can be problematic for ethanol studies because drinking behavior and the reaction to drugs can differ depending on the housing condition of the rodents (Heilig et al. 2016; Ahmed et al. 2020). Therefore, any treatments that reduce ethanol intake in singly housed mice might not be as effective, or may have a different effect altogether, if the subjects are housed socially. Additionally, while some studies have investigated the effect of social-housing on ethanol intake, these studies were still unable to track drinking on an individual level, complicating the assessment of pharmacological interventions on ethanol intake in co-housed animals (Ryabinin and Walcott 2018). As a step forward, advances in radiotracking technology have enabled the study of ethanol intake in group-housed animals at an individual level using Intellicage systems (Smutek et al. 2014; Holgate et al. 2017). However, these cages monitor the number licks for ethanol and water bottles for individual animals that are housed socially, rather than measuring exact amounts of fluid consumed. While the number of licks generally correlates with levels of fluid intake, the exact intake per lick can vary between individuals (Ryabinin and Walcott 2018). In an attempt to address this caveat, a new radiotracking system has been recently developed. The Herdsman-2 (HM2) system (MBRose, Faaborg, Denmark) is designed to track fluid intake at an individual level in socially housed rodents by recording fluid intake (in milliliters), approaches to the fluid bottles and the number of consummatory events, all while also accounting for spillage/evaporation. This system has already been used in two previous studies (Thomsen et al. 2017; Walcott and Ryabinin 2020) assessing ethanol intake in mice and voles, respectively, with promising results.

Thomsen et al. have used the HM2 system to investigate the effects of the glucagon-like peptide 1 receptor agonist exendin- 4 on ethanol intake in socially house male C57BL/6 mice. In this study, treatments were administered per cage, such that all animals in a cage received either the agonist or its vehicle. While this study did provide important findings, the basal ethanol intake in this study was low, and researchers needed to first ethanol-deprive animals to test the effectiveness of exendin-4. More recently, our lab has used this system to assess the effects of OXT on ethanol drinking in socially housed male and female prairie voles (Microtus ochrogaster) (Walcott and Ryabinin 2020). For this study, treatments were administered in a mixed-cage design, such that half of the individuals in each cage received drug treatment while the other half received vehicle. OXT administration decreased ethanol consumption despite the presence of untreated individuals in the same cage. However, the effects of OXT were not specific to ethanol, as this treatment also decreased water intake. These previous studies also did not utilize the HM2 system's ability to examine the temporal dynamics of the treatment effects (only analyzing time-locked cumulative intakes) and its ability to analyze consumption-related behaviors (such as animals' approaches to the fluid bottles and consummatory events). Without these measures, it could be theorized that effects of OXT on ethanol drinking in socially housed animals are mediated by non-specific, for example sedative, effects.

Therefore, the current study used HM2 cages to examine effects of repeated IP OXT administration on two-bottle choice (2BC) ethanol consumption in same-sex groups of male and female C57BL/6J mice. Since this is the first study measuring several ethanol-related consummatory behaviors in unconstrained socially housed male and female mice, we first thoroughly characterized basal ethanol drinking in these animals. After establishing baseline drinking, we administered IP OXT to half of the animals in each cage and examined temporal effects of repeated OXT over 4 days of treatment on ethanol intake and accompanying behaviors.

\section{Methods}

\section{Animals}

C57BL $/ 6 \mathrm{~J}$ ( $n=48$ total, 24 males and 24 females) mice where ordered from The Jackson Laboratory (Bar Harbor, ME, USA) at an age of approximately 2 months upon arrival. Mice were housed in same-sex groups of 4 in standard "shoebox" cages $(18.4 \mathrm{~cm} \mathrm{~W} \times 29.2 \mathrm{~cm} \mathrm{D} \times 12.7 \mathrm{~cm} \mathrm{H})$ 
and on a 12-h light:dark cycle starting at 7 a.m. Mice had access to standard rodent chow and water ad libitum throughout the experiment. Each cage was provided with one nestlet, one pouch of shredded papers, and a $15-\mathrm{cm}$ plastic tube. All procedures were approved by the Oregon Health \& Science University Institutional Animal Care and Use Committee and in accordance with the NIH guidelines. All data were collected prior to March 13, 2020, after which research was restricted due to the COVID-19 pandemic.

\section{Radiofrequency identification tags implantation}

At 6-day post-arrival, mice were implanted with radiofrequency identification tags (RFIDs) to allow monitoring of individual consumption data. Each mouse was weighed, and an RFID chip implanted subcutaneously behind the shoulders (UNO Pico ID Transponder, $7 \mathrm{~mm}$, UNO-PICO-7, Med Associates, Fairfax VT USA) under light isoflurane anesthesia. Mice were returned to the home cage and given 2 days to recover. Mice were then weighed and transferred to the HM2 system with their same-sex cage mates (4 mice/cage).

\section{Apparatus}

Individual drinking and activity was monitored in a social environment using an automated rodent HM2 system. This version of the HM2 system was built to only monitor fluid intake (not food) from two separate bottles. All HM2 apparatuses came equipped with a specialized cage measuring at $48 \mathrm{~cm} \mathrm{~W} \times$ $37.5 \mathrm{~cm} \mathrm{D} \times 21 \mathrm{~cm} \mathrm{H}$. All cages included the same bedding that was provided in home cages, a cotton nestlet, 1 pouch of shredded paper, as well as a 15-cm curved, hard plastic tube. Thus, mice were housed in a relatively "enriched" environment due to the increased space, nesting materials, and plastic tube. The mice had access to fluids in bottles located at the ends of two separate channels. Each 7-cm channel was designed to only allow a single mouse access to the bottle at a time. Channels are equipped with two separate photocells, one at the entrance of the channel and another before the spout. An RFID chip reader is located next to the channel, so that when an individual mouse enters a channel, the photocell detects their presence and the RFID chip reader identifies that individual. Any raw data generated by that mouse is exported by HM2 software and stored on a computer for subsequent analysis. Fluid consumption is measured by weight displacement in the bottle while a mouse is present in the channel. Any lost fluid is caught by a drip tray directly beneath the bottle and spout and is automatically subtracted from the displacement. In addition to spillage, potential evaporation does not affect intake measures, as weight displacement is only measured when an individual is present in the channel. Additional behavioral measures automatically registered by the system were "channel entries", indicating registration of the individual animal when it is breaking the photobeam next to the channel entry, but not the photobeam next to the spout, "drinks", indicating when the registered animal is breaking the photobeam next to the spout and there is a weight displacement of $0.02 \mathrm{~g}$ or greater in the bottle, and "drink size," the volume of solution consumed during each drinking episode.

\section{Training and experimental timeline}

HM2 cages were located in a separate room in close proximity to the mouse colony. The room was kept on reverse 12-hr light cycle (lights off at 11 a.m.). Once transferred to the HM2 cages, mice were given 5 days to habituate to the HM2 system and to the reverse light/dark cycle. The mice remained in HM2 cages on a reverse light cycle through the remainder of the experiment. During this habituation period, mice had access to food and water ad libitum, with water located in bottles at the end of both channels. At the end of the habituation period, one of the water bottles was randomly assigned to be replaced with $3 \%(\mathrm{v} / \mathrm{v})$ ethanol, diluted from $95 \%$ ethanol in autoclaved water, to which mice had continuous access for 2 days. This solution was then replaced with $6 \%(\mathrm{v} / \mathrm{v})$ ethanol, to which mice had continuous access for the remainder of the experiment. While singly housed animals exhibit high levels of intake and high preferences for ethanol concentrations as high as 20\% (Hwa et al. 2011; Nelson et al. 2018; Fulenwider et al. 2019), when housed in a group setting, it has been shown that concentrations greater than $8-10 \%$ can yield extremely low preferences and levels of intake (Holgate et al. 2017; Thomsen et al. 2017). In pilot experiments, we have found that ethanol preference tended to decrease in group-housed mice when the ethanol concentration increased to $8 \%$; therefore, we chose to use $6 \%$ ethanol as the final ethanol concentration presented in the current experiment.

Mice were given 3 days to habituate to the increase in ethanol concentration before proceeding to the treatment experiment. On experimental day 6 , all mice were given a $10 \mathrm{ml} / \mathrm{kg}$ habituation injection of $0.9 \%$ sterile saline at 10:30 a.m. (30 min before the dark cycle began). Following the habituation injection, mice received a daily injection for the next 4 days of either saline or OXT ( $3 \mathrm{mg} / \mathrm{kg}$ or equivalent volume, IP). Two of the four mice in each cage were randomly selected to receive OXT injections and the remaining two to receive vehicle injections. Treatments were administered for four consecutive days. Drinking was analyzed in hourly intervals over the course of 24-h post-treatment. All animals were euthanized $3 \mathrm{~h}$ after their final (fourth) treatment injection on day 10.

\section{Drug}

OXT acetate salt (Bachem, Torrence, CA, USA) was dissolved in sterile $0.9 \%$ saline to $0.3 \mathrm{mg} / \mathrm{ml}$ and injected at 10 $\mathrm{ml} / \mathrm{kg}$. The $3-\mathrm{mg} / \mathrm{kg}$ IP dose was selected based on previous 
dose-response studies showing its selective effects on ethanol intake (Stevenson et al. 2017; Walcott and Ryabinin 2020).

\section{Statistics}

Experimental timeline and apparatus schematic were created using BioRender, and the remaining figures were created using GraphPad Prism software. All statistical analyses were conducted using SPSS software. For analysis of baseline intake and entry data, two-way ANOVAs were conducted using the withinsubjects factor of time and the between-subjects factor of sex. The dependent variables were $\mathrm{g} / \mathrm{kg}$ ethanol intake, $\mathrm{g} / \mathrm{kg}$ water intake, ethanol drinks, water drinks, ethanol drink size, water drink size, ethanol channel entries, or water channel entries. Correlational analyses were also conducted to assess the relationships between $\mathrm{g} / \mathrm{kg}$ ethanol and water intake and number of drinks, drink size, or channel entries. For analysis of intake, drinks, drink sizes, and channel entries following OXT treatment, three-way ANOVAs were conducted using the withinsubjects factor of time and the between-subjects factors of sex and treatment. Where appropriate, planned comparisons or posthoc analyses using Bonferroni correction were conducted.

\section{Results}

\section{Baseline intake, drinks, drink size, and channel entries}

Mice were exposed to a $2 \mathrm{BC}$ procedure with 3 and $6 \%$ ethanol solutions versus water to establish a baseline level of intake (Fig. 1). During baseline drinking, females consumed significantly more $\mathrm{g} / \mathrm{kg}$ ethanol than males $\left(F_{1,46}=5.32, p=0.03\right)$, while baseline water intake did not differ between males and females (Fig. 2a, b). Females also showed higher ethanol preference than males (Figure S1). Hourly analysis of behavior showed that two peaks of ethanol intake developed gradually during days of ethanol exposure (Figure S2). For complete, detailed analysis of these and all other measures in the study, see Supplementary Statistics Table).

The HM2 system also allows for the tracking of number of drinks, drink size, as well as entries into each channel that are not accompanied by consumption of fluid. The difference in number of ethanol drinks between males and females did not reach statistical significance when analyzed at the level of daily intake (Fig. 2c). In contrast, drink size was significantly higher in males $\left(F_{1,46}=11.16, p=0.002\right.$, Fig. $\left.2 \mathrm{e}\right)$. However, when hourly data were analyzed, there was a significantly higher number of drinks in females versus males at several time intervals of ethanol consumption (Figure S3). There were no sex differences in the number of water drinks (Fig. 2d). For additional analyses, see Fig. 2f and Figures S3-6.

While there was no effect of sex on ethanol channel entries, we observed a significant interaction between time and sex
$\left(F_{5,230}=7.65, p<0.0001\right.$, Fig. $\left.2 \mathrm{~g}\right)$. Post-hoc analysis using Bonferroni correction revealed a significantly higher number of entries into the ethanol channel in males versus females on day $1(p=0.01)$. Similarly, while there was no effect of sex on water channel entries, a significant interaction between time and sex was observed $\left(F_{5,230}=9.78, p<0.0001\right.$, Fig. $\left.2 \mathrm{~h}\right)$. Post-hoc analysis using Bonferroni correction revealed a significantly lower number of entries into the water channel in males versus females on day $6(p=0.03$; see Figures S7, S8 for hourly channel entries).

Lastly, the average number of drinks was positively correlated with ethanol ( $p<0.0001$ for males and females, Fig. 3a) and water intake ( $p<0.0001$ for males and females, Fig. 3b). However, drink sizes and the average number of channel entries had no relationship with levels of intake (Fig. 3c-f).

\section{Effects of repeated OXT treatments on ethanol and water intake}

Following 6 days of baseline ethanol intake, mice were treated with either vehicle or OXT over four consecutive days. Since no significant interactions existed between sex and treatment on any of the treatment days, data were collapsed across sex for display purposes only, and all analyses were conducted using the within-subject factor of time and the betweensubject factors of sex and treatment. For data displayed across all factors (including sex), see Figures S9, S10.

While no effect of OXT treatment on ethanol intake was observed on day 1 of treatment, there was a significant interaction observed between time and treatment $\left(F_{23,1012}=2.40, p\right.$ $<0.0001$ ), with planned comparisons analysis revealing that OXT-treated mice consumed significantly less ethanol than vehicle-treated animals at 1 -h $(p<0.01), 2-\mathrm{h}(p<0.0001)$, and 3-h $(p<0.01)$ post-treatment (Fig. 4a).

On the second day of treatment, there was no effect of OXT treatment on ethanol intake, nor an interaction between the factors of time and treatment (Fig. 4b), in contrast to the findings from treatment day 1 .

On the third day of treatment, again, there was no effect of OXT treatment on ethanol intake. However, a significant interaction between time and treatment $\left(F_{23,1012}=2.23, p=\right.$ 0.001 ) was observed, consistent with effects seen on treatment day 1. Planned comparisons analysis revealed that OXT groups consumed significantly less ethanol 2-h $(p<0.01)$ and 3-h $(p<0.05)$ post-treatment, and significantly more ethanol 13-h $(p<0.05)$ post-treatment when compared to vehicle groups (Fig. 4c).

On the fourth day of treatment, a trend-level effect of treatment was observed $\left(F_{1,44}=2.69, p=0.06\right.$, Fig. $\left.4 \mathrm{c}\right)$. There was also a significant interaction between time and treatment $\left(F_{2,88}\right.$ $=6.54, p=0.002$ ), with planned comparisons analysis revealing significantly lower levels of ethanol intake in the OXT versus vehicle group 2 -h post-treatment $(p<0.0001)$. 


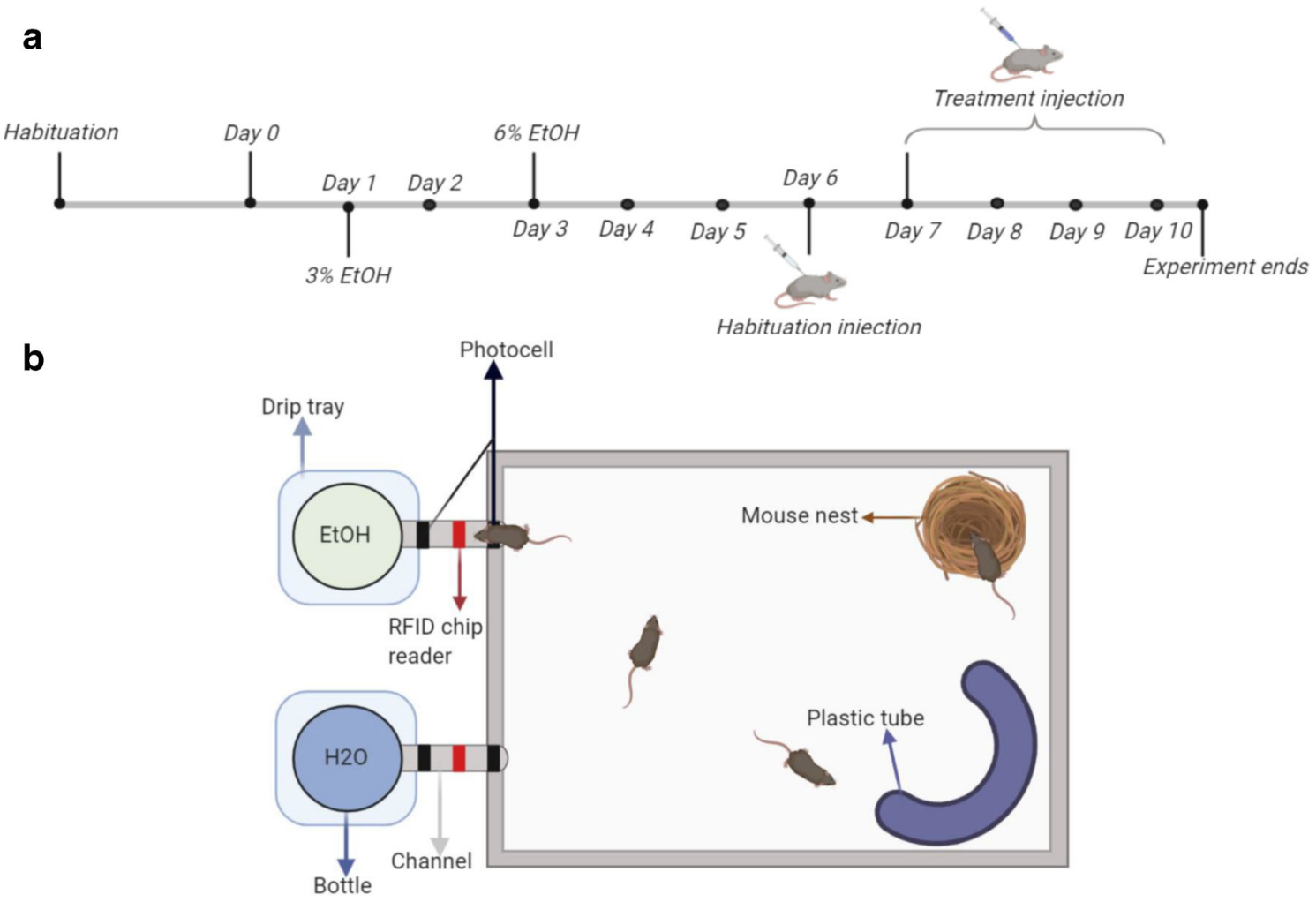

Fig 1 a Experimental timeline. b HM2 schematic

As above, data for water intake were collapsed across sex for display purposes only, and all analyses were conducted using the within-subjects factor of time and the betweensubject factors of sex and treatment. For data displayed across all factors, see Figures S9, S10. OXT treatment had no effect on water intake for all four days of treatment (Figure 5).

\section{Effects of repeated OXT treatments on ethanol drinks and channel entries}

As above, data for number of drinks, drink size, and number of channel entries were collapsed across sex for display purposes only, while all analyses were conducted using the within-subjects factor of time and the between-subject factors of sex and treatment. Interestingly, the effects of OXT on the number of ethanol drinks intake were similar to those observed on ethanol intake.

On the first day of treatment, while no significant effect of OXT treatment on number of ethanol drinks was observed, there was a significant interaction between time and treatment $\left(F_{23,1012}=2.17, p=0.001\right)$, with planned comparisons analysis revealing significantly less ethanol drinks in the OXT versus vehicle group 1-, 2-, and 3-h post-treatment $(p<0.01$ for all timepoints, Fig. 6a).

On the second day of treatment, while there was no effect of OXT treatment on ethanol drinks, a significant interaction between time, sex, and treatment $\left(F_{23,1012}=1.85, p=0.009\right)$ was observed, with planned comparisons analysis revealing a significantly lower number of ethanol drinks in the OXT group 1- and 2-h post-treatment compared with the vehicle group ( $p<0.05$ for both timepoints, Fig. 6b).

As observed on the previous treatment days, on the third day of treatment, there was no main effect of OXT, but there was a significant interaction between time and treatment $\left(F_{23,1012}=1.76, p=0.02\right)$. Planned comparisons analysis revealed a significantly lower number of ethanol drinks in the OXT group 2-h $(p<0.01), 3-\mathrm{h}(p<0.05)$, and 7-h $(p<0.05)$ post-treatment, compared with the vehicle group (Fig. 6c). However, at 13-h post-treatment, OXT-treated mice had a significantly higher number of ethanol drinks than vehicletreated animals $(p<0.05)$.

On the fourth day of treatment, analysis of ethanol drinks revealed a trend-level effect of OXT treatment $\left(F_{1,44}=3.69, p\right.$ $=0.06$, Fig. $6 \mathrm{c})$ and a significant interaction between time and treatment $\left(F_{2,88}=3.85, p=0.03\right)$, with planned comparisons analysis revealing a significantly lower number of drinks in the OXT group 2-h post-treatment compared with the vehicle group $(p<0.01)$.

Since mice do not drink every hour, there was a substantial number of data points missing in the hourly analysis of drink size. Therefore, statistical analysis of hourly drink size was not conducted. However, upon visual inspection, there was never a noticeable decrease in drink size when a decrease in ethanol intake was observed (1-3-h post-treatment, Fig. S11). 
Fig. 2 Baseline ethanol (EtOH) and water $(\mathrm{H} 2 \mathrm{O})$ consumption, drinks, drink size, and channel entries. Darker shaded regions indicate when the mice had access to $3 \% \mathrm{EtOH}$ and the lighter shaded regions indicate when the mice had access to $6 \% \mathrm{EtOH}$. a EtOH intake $(\mathrm{g} / \mathrm{kg})$. b H2O intake $(\mathrm{g} / \mathrm{kg})$. c EtOH drinks. d H2O drinks. e EtOH drink size. f $\mathrm{H} 2 \mathrm{O}$ drink size. g EtOH channel entries. h H2O channel entries. Data expressed as mean $\pm \mathrm{SEM}$. $* p<$ 0.05 , compared to opposite sex. $n$ $=24 / \mathrm{sex}$ a
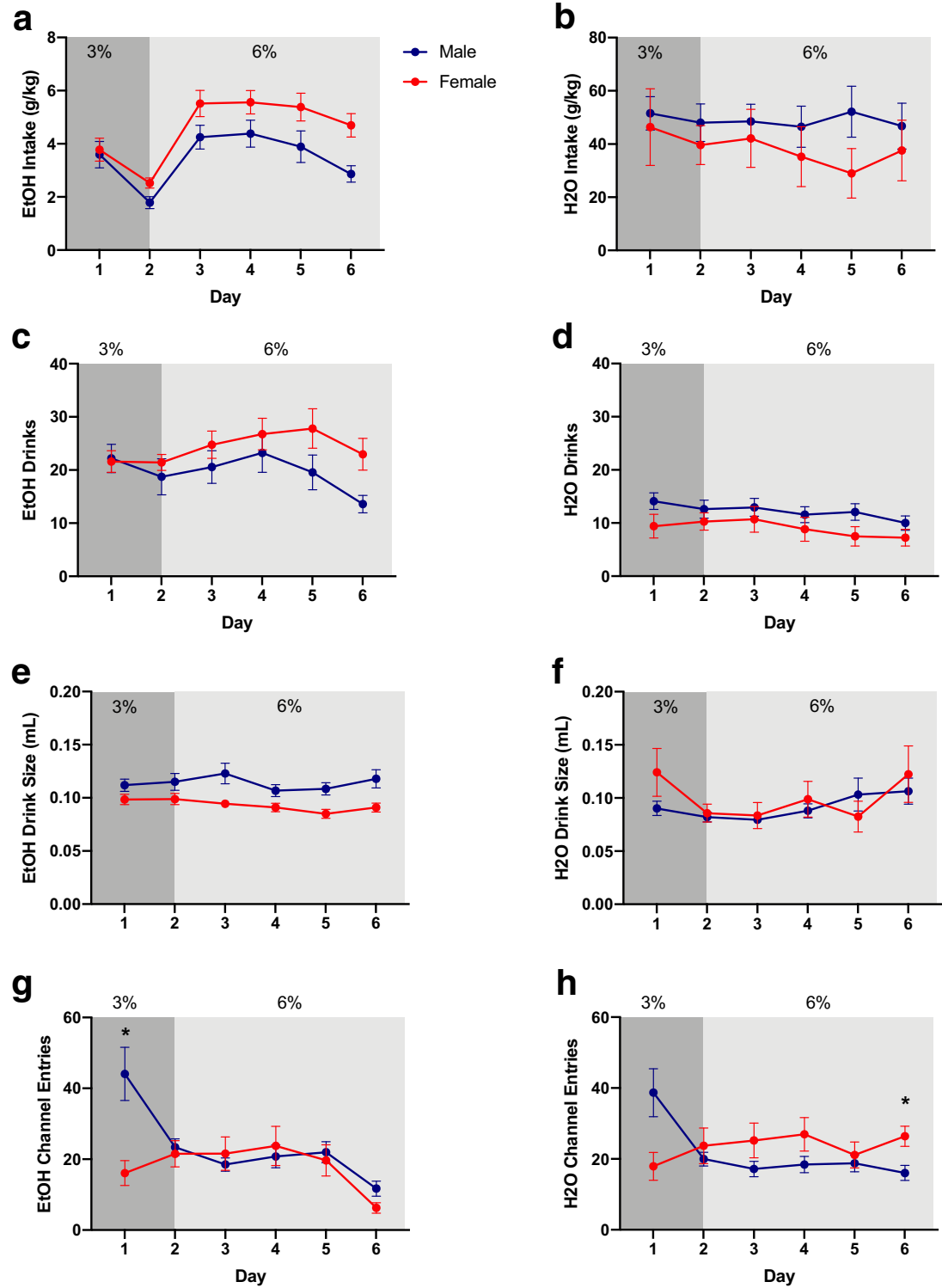

b

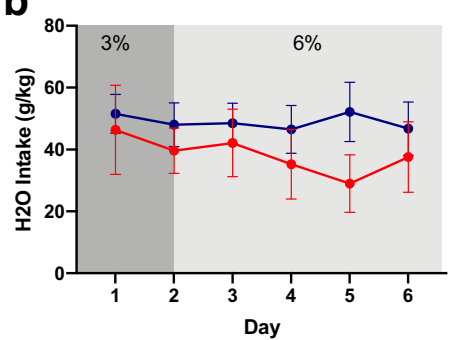

h

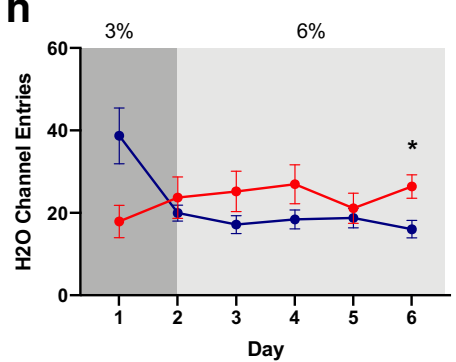

No significant differences in ethanol channel entries between groups were seen for the first three days of treatment (Figure S12; see Supplementary Statistics Table for complete, detailed statistics). On the fourth day of treatment, though, significant interactions between time and treatment $\left(F_{2,88}=4.73, p\right.$ $=0.01)$ and between time, sex, and treatment $\left(F_{2,88}=3.91, p=\right.$ 0.02 ) were observed. Planned comparisons analysis revealed that the OXT group entered the ethanol channel significantly less than the vehicle group 1-h post-treatment $(p<0.05)$.

\section{Effects of repeated OXT treatments on water drinks and channel entries}

No significant effects of OXT on number of water drinks were observed for the first three treatment days (Figures S13). However, on the fourth day of treatment, there was a significantly lower number of water drinks in the OXT group 2-h post-treatment compared with the vehicle group. Similar to ethanol drink size data, statistical analyses of the effect of OXT on water drink size were not conducted because mice do not drink every hour of the day. However, there was never a decrease in drink size at time intervals when a decrease in intake could be expected (1-3-h post-treatment, Figures S14).

For water channel entries, no significant effect of OXT was observed on the first, third, or fourth day of treatment (Figure S15). On the second treatment day, however, there were significantly less water channel entries in the OXT versus vehicle groups at 5 -h and $24-\mathrm{h}$ post-treatment. As mentioned previously, for complete detailed analyses of these all other data, see Supplementary Statistics Table. 
Fig. 3 Baseline correlational analyses. a Average baseline ethanol (EtOH) intake positively correlated with average number of EtOH drinks for males $(r$-squared $=0.78, p<0.0001)$ and females $(r$ squared $=0.78, p<$ $0.0001)$. b Average baseline water $(\mathrm{H} 2 \mathrm{O})$ intake positively correlated with average number of $\mathrm{H} 2 \mathrm{O}$ drinks for males ( $r$-squared $=0.73, p<0.0001)$ and females $(r$ squared $=0.95, p<0.0001)$. c Average baseline EtOH intake was not correlated with EtOH drink size for males $(r$-squared $=$ $0.0004, p=0.93)$ or females $(r$ squared $=0.02, p=0.54)$. $\mathbf{d}$ Average baseline $\mathrm{H} 2 \mathrm{O}$ intake was not correlated with $\mathrm{H} 2 \mathrm{O}$ drink size for males $(r$-squared $<$ $0.0001, p=0.97)$ or females $(r$ squared $=0.0002, p=0.95)$. $\mathbf{e}$ Average baseline EtOH intake was not correlated with average number of EtOH channel entries for males $(r$-squared $=0.07, p=$ $0.21)$ or females $(r$-squared $=$ $0.12, p=0.09)$. f Average baseline $\mathrm{H} 2 \mathrm{O}$ intake was not correlated with average number of $\mathrm{H} 2 \mathrm{O}$ channel entries for males $(r-$ squared $=0.05, p=0.30$ ) or females $(r$-squared $=0.10, p=$ $0.13) . n=24 / \mathrm{sex}$
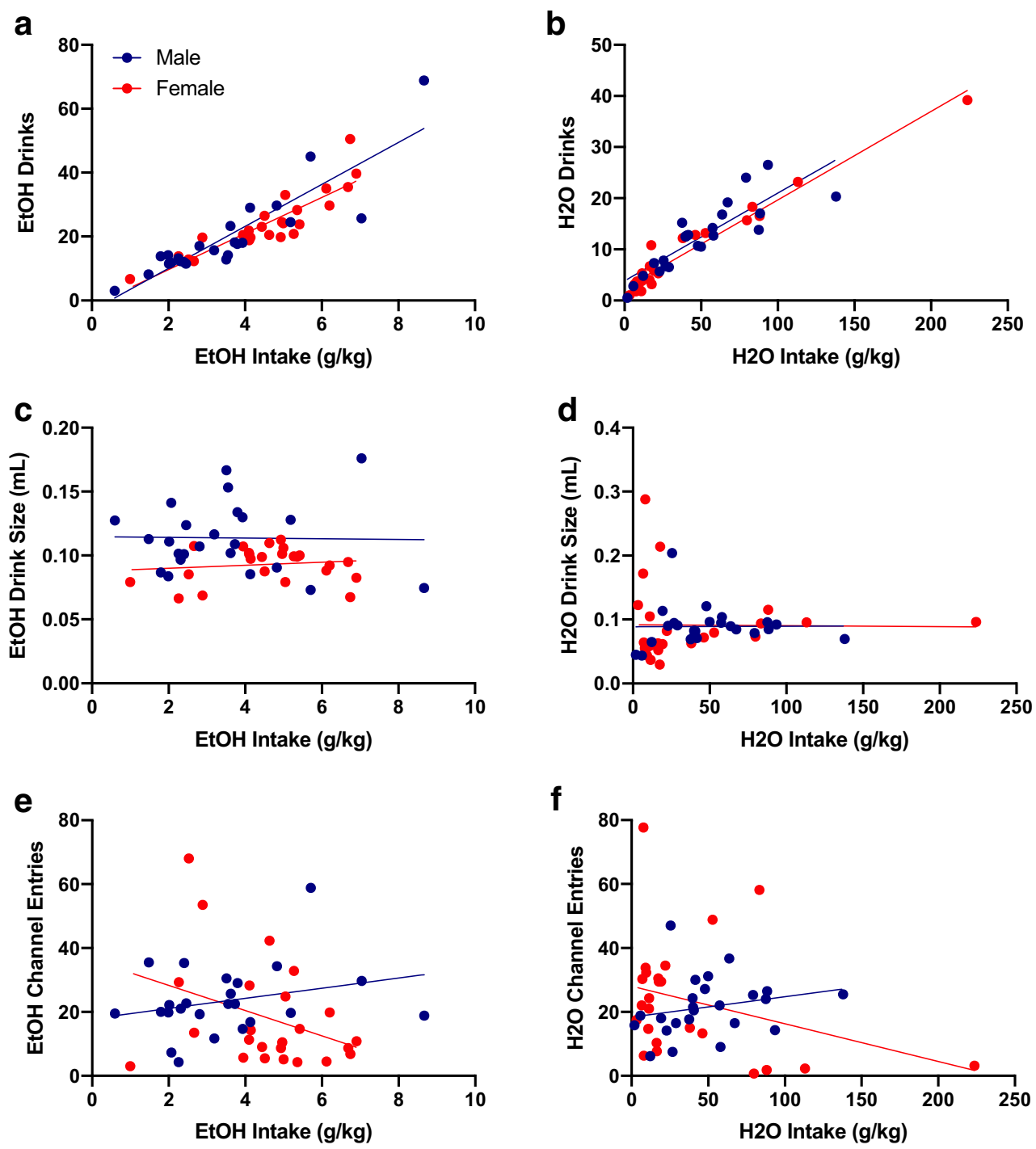

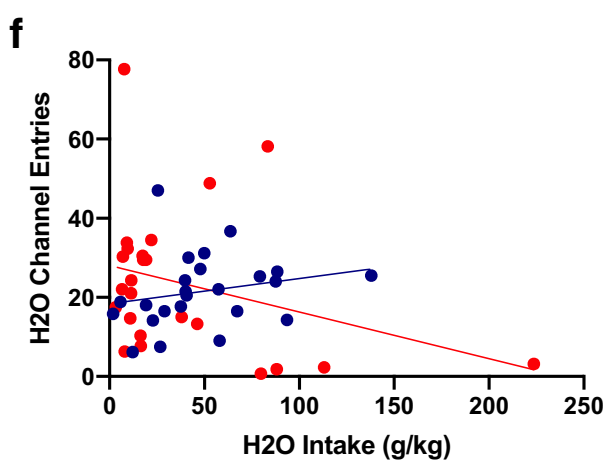

\section{Discussion}

Our studies for the first time characterized detailed ethanol drinking behavior of individual male and female mice housed socially during ethanol $2 \mathrm{BC}$, while also evaluating the temporal effects of OXT on this behavior. We conclude that $\mathrm{g} / \mathrm{kg}$ ethanol intake is most strongly regulated by number of drinks and that the direction and specificity of OXT's effects on ethanol can change over time and with repeated treatment.

\section{Characterization of individual baseline drinking in socially housed male and female B6 mice}

Our study is the second to use HM2 cages to evaluate ethanol drinking in mice. The previous mouse study demonstrated lower baseline intakes and preference than our experiment in the absence of ethanol deprivation (Thomsen et al. 2017). Under these conditions, we also observed lower intakes than most studies using single-housed C57BL/6J mice. This observation is in agreement with previous publications demonstrating that social housing decreases ethanol intake and preference in rodents. However, the levels of ethanol intake and preference observed in our studies are still higher than those reported in previous studies of ethanol drinking in socially housed animals (Smutek et al. 2014; Pradier et al. 2015; Holgate et al. 2017). Specifically, at the $6 \%$ solution (a concentration lower than those used in most studies with socially housed mice), mice in our experiment had higher levels of ethanol intake $(3.9-5.3 \pm 0.24-0.34 \mathrm{~g} / \mathrm{kg})$ and preference $(65-77 \pm 4 \%)$ than those in previous studies. Interestingly, these intakes are comparable with those observed in singlehoused animals using standard $2 \mathrm{BC}$ procedures when similar concentrations are presented (Peters et al. 2013; Marcus et al. 2017; Fulenwider et al. 2019). Therefore, it appears that social housing decreases not ethanol consumption per se, but rather consumption of higher concentrations of ethanol. Since higher concentrations of ethanol are more likely to produce aversive effects, we hypothesize that socially housed mice are more 
Fig. 4 Time-dependent effects of oxytocin on ethanol (EtOH) consumption $(\mathrm{g} / \mathrm{kg})$. Shaded regions of the graph are representative of the dark cycle. a EtOH intake on the first day of treatment. b EtOH intake on the second day of treatment. c EtOH intake on the third and fourth day of treatment. Treatment days three and four were combined for display purposes. Data expressed as mean \pm SEM. $* p<0.05, * p<0.01, * * * p$ $<0.001$, **** $p<0.0001$, compared to vehicle. $n=12 /$ group a OXT treatment 1

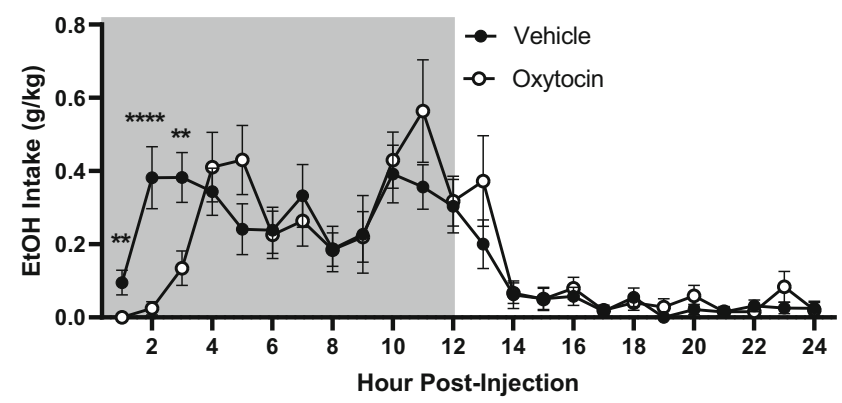

b OXT treatment 2

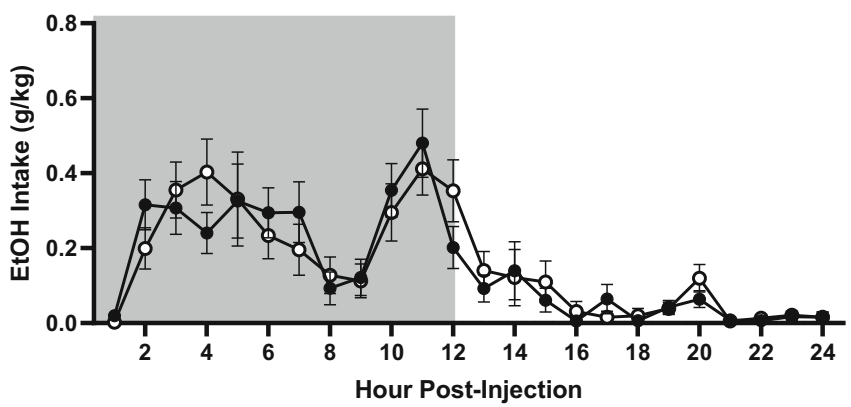

C

OXT treatment 3

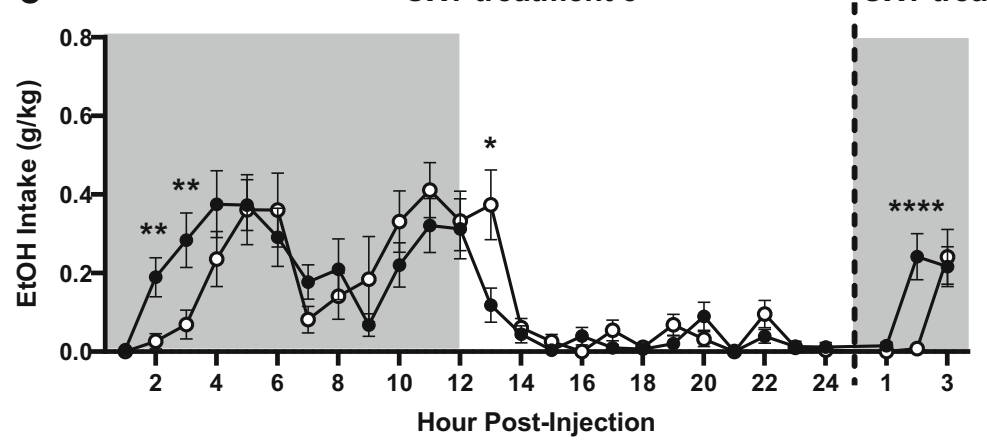

sensitive, or at least cautious, of these aversive effects. To model AUD, future studies will focus on ways to increase ethanol intake by implementing ethanol deprivation and intermittent schedule procedures.

Female mice consumed significantly higher doses of ethanol than males and had a higher ethanol preference (Figures 2A, S1). This observation is consistent with previous studies using single-housed mice (Finn et al. 2004; Melón et al. 2013; Jury et al. 2017; Satta et al. 2018; Fulenwider et al. 2019; Sneddon et al. 2019). Our approach using HM2 cages evaluated whether the higher intake in females was caused by a higher number of drinks taken or other factors, such as a higher volume consumed per drink. Previously, such conclusions could only be made by assessing the number of licks in lickometer approaches or by estimating how much time an animal spends in close proximity to the drinking bottle. The lack of correlation between $\mathrm{g} / \mathrm{kg}$ intake and the number of entries into either the water or ethanol channel indicates that proximity to the drinking bottle is not a good indicator of fluid consumption. In fact, on certain occasions, a significant difference in the number of entries did not correspond to a difference in fluid consumption. This observation was especially noticeable during the first day of baseline drinking behavior, when one of the two bottles was first adulterated with ethanol (see Fig. 2a), although it was also noticeable at other occasions as well (see Fig. $2 \mathrm{~g}$ ). In contrast, the number of ethanol and water drinks were strongly, positively correlated with levels of ethanol and water intake in $\mathrm{g} / \mathrm{kg}$. In fact, to our knowledge, the correlation between the number of drinks and fluid intake in our study was higher than the correlations between number of licks and intake observed in lickometer studies (Ford et al. 2005; Griffin et al. 2007; Ford et al. 2008)

The stronger positive correlation between ethanol intake and number drinks observed here versus the correlation between intake and licks is not surprising, as the number of drinks in the HM2 cages reflects not only the number of an 
Fig. 5 Time-dependent effects of oxytocin on water consumption $(\mathrm{g} / \mathrm{kg})$. Shaded regions of the graph are representative of the dark cycle. a Water intake on the first day of treatment. b Water intake on the second day of treatment. c Water intake on the third and fourth days of treatment Treatment days 3 and 4 were combined for display purposes.

There were no significant differences. $n=12 /$ group
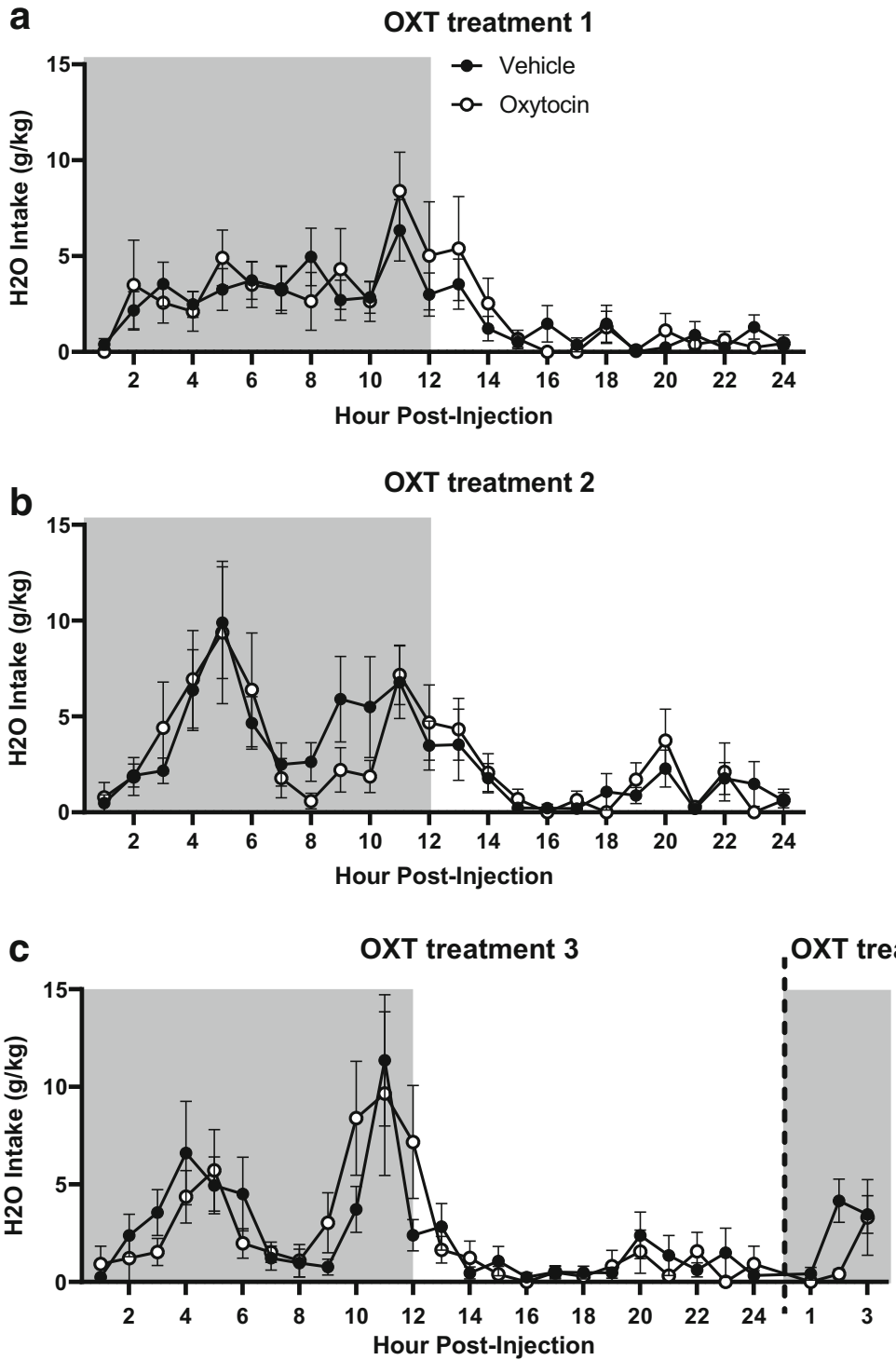

animal's contacts with the spout, but more specifically, the number of contacts that resulted in consumption of fluid. Hourly analysis of behaviors revealed that females reach greater levels of $\mathrm{g} / \mathrm{kg}$ ethanol intake partly due to a higher number of drinks in comparison to males. Interestingly, this higher intake is despite larger sizes of individual drinks in males versus females.

Temporal analysis also indicated that while ethanol drinking showed two main peaks (several hours after lights are off and several hours before lights are on), consumption of water was more evenly distributed across the dark circadian cycle. The two peaks of ethanol intake developed gradually upon ethanol exposure and thus appear not to be a consequence of natural biological activity rhythms. The higher intake of water in males versus females observed towards the end of the dark cycle corresponded to higher number of water drinks taken by males at this time. Overall, these observations indicate that radiofrequency tracking in HM2 cages detects differences in ethanol consumption and accompanying behaviors with high sensitivity, a potentially useful feature for deciphering specific mechanisms by which pharmacological agents regulate ethanol drinking in mice.

\section{Time-dependent effects of OXT on ethanol intake}

Our investigation adds to the accumulating literature demonstrating that OXT can decrease ethanol consumption (McGregor and Bowen 2012; Peters et al. 2013; MacFadyen et al. 2016; King et al. 2017; Stevenson et al. 2017; Hansson et al. 2018). Moreover, it is the second study showing that OXT is capable of decreasing ethanol consumption in mixed-treatment settings, i.e., when only half of the subjects in the group/cage received the treatment. Our laboratory has previously observed this effect in prairie voles using HM2 
Fig. 6 Time-dependent effects of oxytocin on ethanol drinks.

Shaded regions of the graph are representative of the dark cycle. a Ethanol drinks on the first day of treatment. b Ethanol drinks on the second day of treatment. $\mathbf{c}$

Ethanol drinks on the third and fourth day of treatment.

Treatment days 3 and 4 where combined for display purposes. Data expressed as mean \pm SEM. $* p<0.05, * p<0.01$, compared to vehicle. $n=12 /$ group
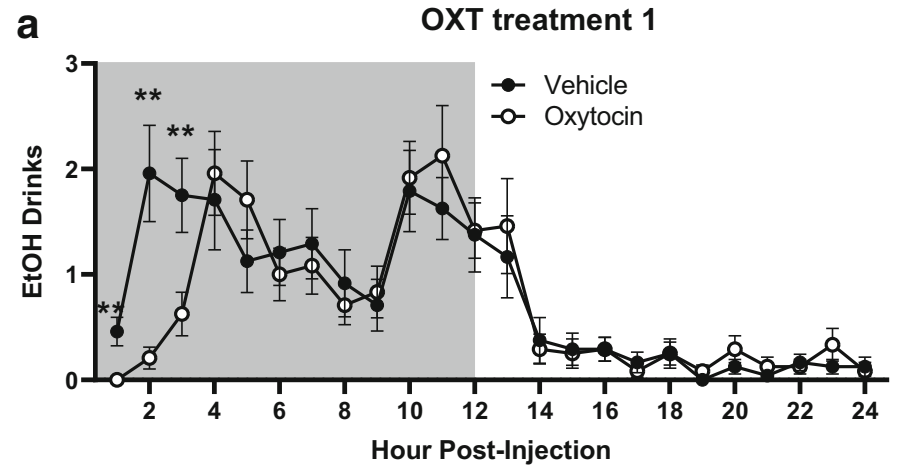

b

OXT treatment 2

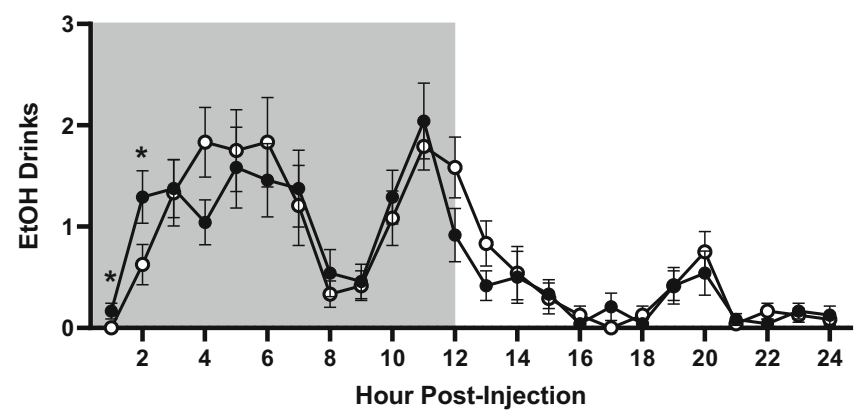

C

OXT treatment 3

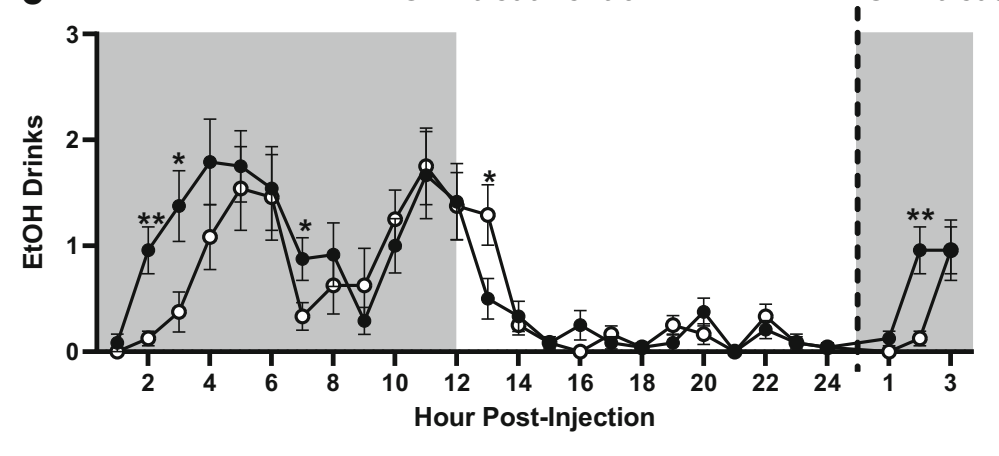

cages (Walcott and Ryabinin 2020). These findings indicate that OXT can decrease ethanol consumption irrespective of potential counteracting influence by untreated subjects.

Interestingly, in the previous HM2 study in prairie voles, OXT did not have selective effects towards ethanol, decreasing both ethanol and water consumption. This is in contrast to our current observation showing that during the first three rounds of OXT treatment, the effects of OXT were specific in decreasing ethanol, but not water, drinking. The lack of OXT's specificity observed in prairie voles could be a species-specific effect due to differences in OXT systems in monogamous versus promiscuous rodent species. Additionally, the prairie voles in the previous experiment had higher baseline levels of ethanol intake compared to those of mice in the current study. Indeed, ethanol consumption in the current model is not an ideal model for AUD, as the levels of ethanol intake were relatively low. However, these findings demonstrate that OXT treatment may also be effective in decreasing modest levels of ethanol consumption, and that this decrease is largely driven by an OXT-induced decrease in the number of drinks taken, demonstrating OXT's ability to decrease the number of drinking occasions.

The $3 \mathrm{mg} / \mathrm{kg}$ dose is substantially higher than doses that are typically used in humans (Pedersen et al. 2013; Lee and Weerts 2016; Hansson et al. 2018). However, this dose is lower than those reported to have suppressed ethanol drinking without affecting water drinking or general locomotor activity in rodent models (Stevenson et al. 2017). The higher doses of OXT could theoretically produce non-selective effects by acting at not only OXT, but also vasopressin receptors. However, our findings argue against this possibility. During the first three treatment cycles, OXT selectively decreased ethanol intake and number of ethanol drinks, but not water intake or the number of water drinks. Furthermore, regardless of the high dose of OXT used in our study, clearance of this peptide from the blood is rapid, such that it is 
expected to be completely eliminated within $1 \mathrm{~h}$ of treatment (Neumann et al. 2013; Lee et al. 2018).

Additionally, controversy exists in the field regarding the pharmacodynamics of IP-administered OXT effects on behavior. Passive blood brain penetrance of OXT is clearly low (Ermisch et al. 1985; Macdonald and Feifel 2013; Neumann et al. 2013; Lee et al. 2018). Nevertheless, active transport of OXT by Receptor for Advanced Glycation End-products (RAGE) to the brain has been recently observed in mice (Yamamoto et al. 2019; Yamamoto and Higashida 2020). Effects of OXT could also be enhanced by feed-forward mechanisms where peripheral OXT stimulates central actions of OXT (Neumann et al. 2013). Such feed-forward mechanisms could explain effects of peripheral mechanisms at times when the peptide is eliminated from the bloodstream.

Perhaps the most interesting and potentially worrisome effect of OXT was the variability in treatment efficacy day-today. For example, the effect of OXT on ethanol intake was much weaker on day 2 of treatment in comparison to the previous and subsequent treatment days. Additionally, although OXT selectively decreased ethanol intake within the first several hours of drinking during first three treatment days, a rebound in ethanol intake was observed 13-h post-treatment during the second and third days of treatment, in which OXTtreated mice consumed more ethanol than vehicle-treated controls (see Figs. 4c and 6c). Such inconsistent effects over time were not observed in the previous prairie vole study in the HM2 cages where the same dose of OXT continued to be effective with repeated treatments (Walcott and Ryabinin 2020). Since the ethanol intake in the prairie vole study was higher than here, and yet more consistent effects of treatment were observed there, we hypothesize that mice are less sensitive to OXT than prairie voles. Interestingly however, although in the present study OXT was observed to have specific effects on ethanol intake during the first three treatments, its effects during the fourth treatment appeared to become less selective. In particular, following the fourth treatment, OXT decreased not only ethanol intake and the number of ethanol drinks, but also water intake, the number of water drinks, and the number of entries into the ethanol channel, suggesting that on the fourth day of treatment, OXT may have decreased ethanol intake through off-target effects, such as sedation or general motivation to consume any fluid.

Differential effects of OXT in acute versus repeated administration on various behaviors have been previously noted (Szabó et al. 1985; Huang et al. 2014; Lee et al. 2016; Benner et al. 2018), with the majority of studies reporting that OXT decreases in efficacy with repeated treatments. In contrast, our findings suggest that OXT's effects change from more selective to non-selective and suggest sensitization in the systems affected by OXT. This sensitization could occur in OXT or vasopressin receptors, or could even involve signal transduction mechanisms affected by activation of these receptors. The role of the vasopressin system in these offtarget consummatory effects is of particularly high relevance given this system's established importance in regulating water balance (Danziger and Zeidel 2015; Bankir et al. 2017). Additionally, while we did not directly measure the effects of OXT treatment on food consumption, acute OXT treatment can significantly decrease food intake (Arletti et al. 1989; Olson et al. 1991; Ott et al. 2013). Thus, the effect of repeated, IP OXT administration on food intake and on palatable, ethanol-free, sucrose solutions will be examined in future experiments.

\section{Conclusions}

Taken together, our studies are the first to provide detailed analysis of individual ethanol intake in socially housed male and female mice using the HM2 system. This development promises improved preclinical testing of potential medications for AUD treatment. Moreover, while confirming that OXT can decrease ethanol consumption, we demonstrate for the first time that this treatment can have inconsistent effects as well as potential non-selective effects following repeated administration. Therefore, we conclude that although the OXT system remains a promising target for the treatment of AUD, more studies are needed to assess potential negative consequences of this treatment, especially following repeated treatment and that development of better OXT receptor agonists could be a promising future avenue for research in this area.

Supplementary Information The online version contains supplementary material available at https://doi.org/10.1007/s00213-020-05741-3.

Funding This work was supported by NIH Grants RO1 AA019793 and T32 AA007468.

\section{Compliance with ethical standards}

Conflict of interest The authors declare that they have no conflict of interest.

\section{References}

Ahmed SH, Badiani A, Miczek KA, Müller CP (2020) Nonpharmacological factors that determine drug use and addiction. Neurosci Biobehav Rev 110:3-27

Akbar M, Egli M, Cho YE, Song BJ, Noronha A (2018) Medications for alcohol use disorders: An overview. Pharmacol Ther 185:64-85

Arletti R, Benelli A, Bertolini A (1989) Influence of oxytocin on feeding behavior in the rat. Peptides 10(1):89-93

Bankir L, Bichet DG, Morgenthaler NG (2017) Vasopressin: physiology, assessment and osmosensation. J Intern Med 282(4):284-297

Becker HC (2013) Animal models of excessive alcohol consumption in rodents. Curr Top Behav Neurosci 13:355-377 
Benner S, Aoki Y, Watanabe T, Endo N, Abe O, Kuroda M, Kuwabara H, Kawakubo Y, Takao H, Kunimatsu A, Kasai K, Bito H, Kakeyama M, Yamasue H (2018) Neurochemical evidence for differential effects of acute and repeated oxytocin administration. Mol Psychiatry

Bowen MT, Carson DS, Spiro A, Arnold JC, McGregor IS (2011) Adolescent oxytocin exposure causes persistent reductions in anxiety and alcohol consumption and enhances sociability in rats. PLoS One 6(11):e27237

Crabbe JC, Harris RA, Koob GF (2011) Preclinical studies of alcohol binge drinking. Ann N Y Acad Sci 1216:24-40

Danziger J, Zeidel ML (2015) Osmotic homeostasis. Clin J Am Soc Nephrol 10(5):852-862

Ermisch A, Ruhle HJ, Landgraf R, Hess J (1985) Blood-brain barrier and peptides. J Cereb Blood Flow Metab 5(3):350-357

Finn DA, Sinnott RS, Ford MM, Long SL, Tanchuck MA, Phillips TJ (2004) Sex differences in the effect of ethanol injection and consumption on brain allopregnanolone levels in C57BL/6 mice. Neuroscience 123(4):813-819

Ford MM, Beckley EH, Nickel JD, Eddy S, Finn DA (2008) Ethanol intake patterns in female mice: influence of allopregnanolone and the inhibition of its synthesis. Drug Alcohol Depend 97(1-2):73-85

Ford MM, Nickel JD, Phillips TJ, Finn DA (2005) Neurosteroid modulators of $\operatorname{GABA}(\mathrm{A})$ receptors differentially modulate Ethanol intake patterns in male C57BL/6J mice. Alcohol Clin Exp Res 29(9):1630 1640

Fulenwider HD, Nennig SE, Price ME, Hafeez H, Schank JR (2019) Sex differences in aversion-Resistant Ethanol Intake in Mice. Alcohol Alcohol 54(4):345-352

Griffin WC 3rd, Middaugh LD, Becker HC (2007) Voluntary ethanol drinking in mice and ethanol concentrations in the nucleus accumbens. Brain Res 1138:208-213

Hansson AC, Koopmann A, Uhrig S, Buhler S, Domi E, Kiessling E, Ciccocioppo R, Froemke RC, Grinevich V, Kiefer F, Sommer WH, Vollstadt-Klein S, Spanagel R (2018) Oxytocin Reduces Alcohol Cue-Reactivity in Alcohol-Dependent Rats and Humans. Neuropsychopharmacology 43(6):1235-1246

Heilig M, Epstein DH, Nader MA, Shaham Y (2016) Time to connect: bringing social context into addiction neuroscience. Nat Rev Neurosci 17(9):592-599

Holgate JY, Garcia H, Chatterjee S, Bartlett SE (2017) Social and environmental enrichment has different effects on ethanol and sucrose consumption in mice. Brain Behav 7(8):e00767

Huang H, Michetti C, Busnelli M, Manago F, Sannino S, Scheggia D, Giancardo L, Sona D, Murino V, Chini B, Scattoni ML, Papaleo F (2014) Chronic and acute intranasal oxytocin produce divergent social effects in mice. Neuropsychopharmacology 39(5):1102-1114

Huynh N, Arabian NM, Asatryan L, Davies DL (2019) Murine drinking models in the development of pharmacotherapies for alcoholism: drinking in the dark and two-bottle choice. J Vis Exp (143)

Hwa LS, Chu A, Levinson SA, Kayyali TM, DeBold JF, Miczek KA (2011) Persistent escalation of alcohol drinking in C57BL/6J mice with intermittent access to $20 \%$ ethanol. Alcohol Clin Exp Res 35(11):1938-1947

Jury NJ, DiBerto JF, Kash TL, Holmes A (2017) Sex differences in the behavioral sequelae of chronic ethanol exposure. Alcohol 58:53-60

King CE, Griffin WC, Luderman LN, Kates MM, McGinty JF, Becker HC (2017) Oxytocin Reduces Ethanol Self-Administration in Mice. Alcohol Clin Exp Res 41(5):955-964

Koob GF, Volkow ND (2016) Neurobiology of addiction: a neurocircuitry analysis. Lancet Psychiatry 3(8):760-773

Kramer KM, Choe C, Carter CS, Cushing BS (2006) Developmental effects of oxytocin on neural activation and neuropeptide release in response to social stimuli. Horm Behav 49(2):206-214

Lee HJ, Macbeth AH, Pagani JH, Young WS 3rd (2009) Oxytocin: the great facilitator of life. Prog Neurobiol 88(2):127-151
Lee MR, Rohn MC, Tanda G, Leggio L (2016) Targeting the Oxytocin System to Treat Addictive Disorders: Rationale and Progress to Date. CNS Drugs 30(2):109-123

Lee MR, Scheidweiler KB, Diao XX, Akhlaghi F, Cummins A, Huestis MA, Leggio L, Averbeck BB (2018) Oxytocin by intranasal and intravenous routes reaches the cerebrospinal fluid in rhesus macaques: determination using a novel oxytocin assay. Mol Psychiatry 23(1):115-122

Lee MR, Weerts EM (2016) Oxytocin for the treatment of drug and alcohol use disorders. Behav Pharmacol 27(8):640-648

Macdonald K, Feifel D (2013) Helping oxytocin deliver: considerations in the development of oxytocin-based therapeutics for brain disorders. Front Neurosci 7:35

MacFadyen K, Loveless R, DeLucca B, Wardley K, Deogan S, Thomas C, Peris J (2016) Peripheral oxytocin administration reduces ethanol consumption in rats. Pharmacol Biochem Behav 140:27-32

Marcus DJ, Henderson-Redmond AN, Gonek M, Zee ML, Farnsworth JC, Amin RA, Andrews MJ, Davis BJ, Mackie K, Morgan DJ (2017) Mice expressing a "hyper-sensitive" form of the CB1 cannabinoid receptor (CB1) show modestly enhanced alcohol preference and consumption. PLoS One 12(4):e0174826

McGregor IS, Bowen MT (2012) Breaking the loop: oxytocin as a potential treatment for drug addiction. Horm Behav 61(3):331-339

Melón LC, Wray KN, Moore EM, Boehm SL 2nd (2013) Sex and age differences in heavy binge drinking and its effects on alcohol responsivity following abstinence. Pharmacol Biochem Behav 104:177-187

National Institute on Alcohol Abuse and Alcoholism (2014) Treatment for alcohol problems: finding and getting help

National Institute on Alcohol Abuse and Alcoholism (2018) Alcohol facts and statistics, 2020, from https://www.niaaa.nih.gov/ publications/brochures-and-fact-sheets/alcohol-facts-and-statistics

Nelson BS, Sequeira MK, Schank JR (2018) Bidirectional relationship between alcohol intake and sensitivity to social defeat: association with Tacr1 and Avp expression. Addict Biol 23(1):142-153

Neumann ID, Maloumby R, Beiderbeck DI, Lukas M, Landgraf R (2013) Increased brain and plasma oxytocin after nasal and peripheral administration in rats and mice. Psychoneuroendocrinology 38(10): 1985-1993

Olson BR, Drutarosky MD, Chow MS, Hruby VJ, Stricker EM, Verbalis JG (1991) Oxytocin and an oxytocin agonist administered centrally decrease food intake in rats. Peptides 12(1):113-118

Ott V, Finlayson G, Lehnert H, Heitmann B, Heinrichs M, Born J, Hallschmid M (2013) Oxytocin reduces reward-driven food intake in humans. Diabetes 62(10):3418-3425

Pedersen CA, Smedley KL, Leserman J, Jarskog LF, Rau SW, KampovPolevoi A, Casey RL, Fender T, Garbutt JC (2013) Intranasal oxytocin blocks alcohol withdrawal in human subjects. Alcohol Clin Exp Res 37(3):484-489

Peters S, Slattery DA, Flor PJ, Neumann ID, Reber SO (2013) Differential effects of baclofen and oxytocin on the increased ethanol consumption following chronic psychosocial stress in mice. Addict Biol 18(1):66-77

Pradier B, Erxlebe E, Markert A, Rácz I (2015) Interaction of cannabinoid receptor 2 and social environment modulates chronic alcohol consumption. Behav Brain Res 287:163-171

Ryabinin AE, Walcott AT (2018) Assessing social alcohol drinking in rodent models: are we there yet. Int Rev Neurobiol 140:33-51

Satta R, Hilderbrand ER, Lasek AW (2018) Ovarian hormones contribute to high levels of binge-like drinking by female mice. Alcohol Clin Exp Res 42(2):286-294

Smutek M, Turbasa M, Sikora M, Piechota M, Zajdel J, Przewlocki R, Parkitna JR (2014) A model of alcohol drinking under an intermittent access schedule using group-housed mice. PLoS One 9(5): e96787 
Sneddon EA, White RD, Radke AK (2019) Sex differences in binge-like and aversion-resistant alcohol drinking in $\mathrm{C} 57 \mathrm{BL} / 6 \mathrm{~J}$ mice. Alcohol Clin Exp Res 43(2):243-249

Stevenson JR, Wenner SM, Freestone DM, Romaine CC, Parian MC, Christian SM, Bohidar AE, Ndem JR, Vogel IR, O'Kane CM (2017) Oxytocin reduces alcohol consumption in prairie voles. Physiol Behav 179:411-421

Swift RM, Aston ER (2015) Pharmacotherapy for alcohol use disorder: current and emerging therapies. Harv Rev Psychiatry 23(2):122133

Szabó G, Kovács GL, Székeli S, Telegdy G (1985) The effects of neurohypophyseal hormones on tolerance to the hypothermic effect of ethanol. Alcohol 2(4):567-574

Thomsen M, Dencker D, Wortwein G, Weikop P, Egecioglu E, Jerlhag E, Fink-Jensen A, Molander A (2017) The glucagon-like peptide 1 receptor agonist Exendin-4 decreases relapse-like drinking in socially housed mice. Pharmacol Biochem Behav 160:14-20

Tunstall BJ, Kirson D, Zallar LJ, McConnell SA, Vendruscolo JCM, Ho CP, Oleata CS, Khom S, Manning M, Lee MR, Leggio L, Koob GF, Roberto M, Vendruscolo LF (2019) Oxytocin blocks enhanced motivation for alcohol in alcohol dependence and blocks alcohol effects on GABAergic transmission in the central amygdala. PLoS Biol 17(4):e2006421
Walcott AT, Ryabinin AE (2020) Assessing effects of oxytocin on alcohol consumption in socially housed prairie voles using radio frequency tracking. Addict Biol e12893

Williams JR, Insel TR, Harbaugh CR, Carter CS (1994) Oxytocin administered centrally facilitates formation of a partner preference in female prairie voles (Microtus ochrogaster). J Neuroendocrinol 6(3): 247-250

Yamamoto Y, Higashida H (2020) RAGE regulates oxytocin transport into the brain. Commun Biol 3(1):70

Yamamoto Y, Liang M, Munesue S, Deguchi K, Harashima A, Furuhara K, Yuhi T, Zhong J, Akther S, Goto H, Eguchi Y, Kitao Y, Hori O, Shiraishi Y, Ozaki N, Shimizu Y, Kamide T, Yoshikawa A, Hayashi Y, Nakada M, Lopatina O, Gerasimenko M, Komleva Y, Malinovskaya N, Salmina AB, Asano M, Nishimori K, Shoelson SE, Yamamoto H, Higashida H (2019) Vascular RAGE transports oxytocin into the brain to elicit its maternal bonding behaviour in mice. Commun Biol 2:76

Publisher's note Springer Nature remains neutral with regard to jurisdictional claims in published maps and institutional affiliations. 